\title{
Application of 3D heat diffusion to detect embedded empty cracks
}

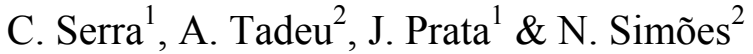 \\ ${ }^{1}$ ITeCons - Institute of Research and Technological Development in \\ Construction Sciences, Coimbra, Portugal \\ ${ }^{2}$ Department of Civil Engineering, University of Coimbra, Portugal
}

\begin{abstract}
This paper presents a three-dimensional boundary element model (BEM), formulated in the frequency domain, to simulate the heat diffusion by conduction that occurs in the vicinity of a three-dimensional crack. The crack is assumed to have null thickness and does not allow diffusion of energy, so the heat fluxes are null along its boundary. The crack is assumed to be embedded in an unbounded medium. The boundary element formulation is written in terms of normalderivative integral equations (known as TBEM) in order to handle null thickness cracks. The resulting hypersingular integrals are solved analytically.

After verifying the TBEM results by means of known analytical solutions for cylindrical inclusions, the applicability of the proposed methodology is shown by simulating the heat diffusion generated by a point heat source placed in the vicinity of a crack. The size of the crack, its orientation and the position of the source are some of the variables that are analyzed to define features that may be used in non-destructive testing by infrared thermography.

Keywords: transient heat conduction, 3D sources, infrared thermography.
\end{abstract}

\section{Introduction}

Heat and moisture diffusion is sensitive to the presence of defects, hence thermal pattern images of building elements collected via Infrared Thermography (IRT) have proven to be useful in detecting heat losses, missing or damaged insulation, thermal bridges, air leakages and excess moisture $[1,2]$ and for testing the integrity of composites [3,4]. IRT is mostly used as a qualitative nondestructive testing technique, however solving inverse heat conduction problems 
in transient regime studied via numerical simulation of heat diffusion will lead to a better understanding of thermo graphic data results and ultimately result in better identification and characterization of defects in building elements [5].

Numerical modeling of heat transfer and diffusion in the presence of defects along with sophisticated techniques such as IRT systems may be employed as a non-destructive technique for detecting the presence and evaluating the extension of existing defects. The three dimensional nature of such defects combined with the need to simulate heat transfer and diffusion phenomena in transient regime presents a challenge for researchers. The present paper presents a model motivated by an interest in assessing the potential of IRT use in the presence of defects with specific geometries and depths.

Within the available numerical methods for homogeneous unbounded or semi-infinite systems modelling, the Boundary Element Method (BEM) [6] is considered to be one of the most adequate tools for this kind of problem since it automatically satisfies far field conditions and only requires the discretization of the inclusions boundaries. However, one major drawback of the BEM is that it can only be applied to more general geometries and media when the relevant fundamental solution is known, which may not always be possible. In addition, it is well known that when using BEM the boundary integrals may become singular or nearly singular depending on the distance between the source point and the node being integrated. Also, it is known that when the thickness of the heterogeneity being modelled tends towards zero, such as in the case of delaminations, cracks or thin defects, the conventional direct BEM degenerates and becomes inaccurate and is no longer a valid basis for numerical modelling. Various techniques have been proposed to overcome this such as the use of Dual Boundary Element Method (DBEM) which leads to hypersingular integrals.

Different approaches have been proposed to deal with hypersingular integrals that arise in DBEM [7]. Solutions for specific 2D problems may be found in Cruse [8], Sládek and Sládek [9], Mendes and Tadeu [10]. For 3D problems, singular integrals are mostly solved using numerical schemes based on Gaussian integration schemes. However, as the accuracy of the BEM is highly dependent on the precision of those integrals, some researchers are looking for sophisticated approaches to solve specific problems [1].

In the sections that follow, a frequency domain boundary element formulation is proposed (the normal-derivative integral equations - TBEM) to simulate the heat diffusion in the vicinity of a 3D crack. Analytical solutions are used to solve the hypersingular integrals when the element being integrated is the loaded one. The numerical model is then verified by comparing its results with known closed-form solutions. Finally, the methodology to obtain time-domain responses from frequency-domain calculations is described, followed by a set of numerical applications to illustrate the applicability and usefulness of the proposed approach in the analysis of several test cases, simulating the heat diffusion generated by a point heat source placed in the vicinity of a crack. These applications mainly focus on the effect of the position, size and orientation of the crack, defining features that may be used in IRT. 


\section{Problem definition}

This work simulates the three-dimensional heat diffusion in the vicinity of a 3D crack, generated by a point heat source, as illustrated in Figure 1. The medium is an unbounded spatially uniform solid medium of density $\rho$, thermal conductivity $\lambda$ and specific heat $c$. The crack, with surface $S$, is assumed to be thin.



Figure 1: Three-dimensional geometry of the problem.

This system is subjected to a point heat source placed at $\left(x_{s}, y_{s} z_{s}\right)$,

$$
\hat{f}(x, y, z, t)=\delta\left(x-x_{s}\right) \delta\left(y-y_{s}\right) \delta\left(z-z_{s}\right) \mathrm{e}^{\mathrm{i} \omega t},
$$

where $\delta\left(x-x_{s}\right), \delta\left(y-y_{s}\right)$ and $\delta\left(z-z_{s}\right)$ are Dirac-delta functions, and $\omega$ is the frequency of the source. The response of this heat source can be expressed by

$$
T_{\text {inc }}\left(x, y, z, x_{s}, y_{s}, z_{s}, \omega\right)=\frac{\mathrm{e}^{-\mathrm{i} \sqrt{-\frac{\mathrm{i} \omega}{K}} r_{0}}}{2 \lambda r_{0}},
$$

in which $K$ is the thermal diffusivity defined by $\frac{\lambda}{\rho c}, \omega$ is the oscillating frequency, $\mathrm{i}=\sqrt{-1}$ and $r_{0}=\sqrt{\left(x-x_{s}\right)^{2}+\left(y-y_{s}\right)^{2}+\left(z-z_{s}\right)^{2}}$.

\section{The normal-derivative integral equation (TBEM)}

The transient heat transfer by conduction is governed by the diffusion equation:

$$
\left(\frac{\partial^{2}}{\partial x^{2}}+\frac{\partial^{2}}{\partial y^{2}}+\frac{\partial^{2}}{\partial z^{2}}\right) T(\boldsymbol{x}, \omega)+\left(k_{c}\right)^{2} T(\boldsymbol{x}, \omega)=0
$$

in which $x=(x, y, z)$ and $k_{c}=\sqrt{\frac{-\mathrm{i} \omega}{K}}$.

The classical boundary integral equation, formulated in the frequency domain, is:

$$
b T\left(\boldsymbol{x}_{0}, \omega\right)=-\int_{S} H\left(\boldsymbol{x}, \mathbf{n}_{n 1}, \boldsymbol{x}_{0}, \omega\right) T(\boldsymbol{x}, \omega) \mathrm{d} s+T_{\text {inc }}\left(\boldsymbol{x}_{0}, \boldsymbol{x}_{s}, \omega\right)
$$

where $H$ are the fundamental solutions (Green's functions) for the heat flux (q), at a point $\boldsymbol{x}=(x, y, z)$ on the boundary $S$, due to a virtual point heat source at 
$\boldsymbol{x}_{0}=\left(x_{0}, y_{0}, z_{0}\right) ; \mathbf{n}_{n 1}$ represents the unit outward normal along the boundary $S$, at $\boldsymbol{x}=(x, y, z) ; b$ is a constant defined by the shape of the boundary, taking the value $1 / 2$ if $\boldsymbol{x}_{0}=\left(x_{0}, y_{0}, z_{0}\right) \in S$, and 1 otherwise.

The required Green's functions for heat flux in an unbounded medium, in Cartesian coordinates, are given by:

$$
H\left(\boldsymbol{x}, \mathbf{n}_{n 1}, \boldsymbol{x}_{0}, \omega\right)=\frac{\mathrm{e}^{-\mathrm{i} k_{c} r}\left(-\mathrm{i} k_{c} r-1\right)}{4 \lambda \pi r^{2}} \frac{\partial r}{\partial \mathbf{n}_{n 1}},
$$

with $r=\sqrt{\left(x-x_{0}\right)^{2}+\left(y-y_{0}\right)^{2}+\left(z-z_{0}\right)^{2}}$

The normal-derivative integral equation can be derived by applying the gradient operator to the boundary integral Eqn (4), which can be seen as assuming the existence of dipole heat sources. When the boundary of the inclusion is loaded with dipoles (dynamic doublets) the required integral equations can be expressed as:

$$
a T\left(\boldsymbol{x}_{0}, \omega\right)=-\int_{S} \bar{H}\left(\boldsymbol{x}, \mathbf{n}_{n 1}, \mathbf{n}_{n 2}, \boldsymbol{x}_{0}, \omega\right) T(\boldsymbol{x}, \omega) \mathrm{ds}+\bar{T}_{i n c}\left(\boldsymbol{x}_{0}, \mathbf{n}_{n 2}, \boldsymbol{x}_{s}, \omega\right),
$$

The Green's functions $\bar{H}$ are defined by applying the gradient operator to $H$, which can be seen as the derivatives of these former Green's functions, to obtain heat fluxes. In these equations, $\mathbf{n}_{n 2}$ is the unit outward normal to the boundary $S$ at the collocation points $\boldsymbol{x}_{0}=\left(x_{0}, y_{0}, z_{0}\right)$, defined by the vector $\mathbf{n}_{n 2}$. In this equation, the factor $a$ is null for piecewise planar boundary elements.

The required three-dimensional Green's functions for an unbounded space are now defined as:

$$
\begin{gathered}
\bar{H}\left(\boldsymbol{x}, \mathbf{n}_{n 1}, \mathbf{n}_{n 2}, \boldsymbol{x}_{0}, \omega\right)=\frac{\partial H}{\partial x} \frac{\partial x}{\partial \mathbf{n}_{n 2}}+\frac{\partial H}{\partial y} \frac{\partial y}{\partial \mathbf{n}_{n 2}}+\frac{\partial H}{\partial z} \frac{\partial z}{\partial \mathbf{n}_{n 2}}, \\
\frac{\partial H}{\partial x}\left(\boldsymbol{x}, \mathbf{n}_{n 1}, \mathbf{n}_{n 2}, \boldsymbol{x}_{0}, \omega\right)=\frac{1}{4 \pi \lambda}\left\{A\left[\left(\frac{\partial r}{\partial x}\right)^{2} \frac{\partial x}{\partial \mathbf{n}_{n 1}}+\frac{\partial r}{\partial x} \frac{\partial r}{\partial y} \frac{\partial y}{\partial \mathbf{n}_{n 1}}+\frac{\partial r}{\partial x} \frac{\partial r}{\partial z} \frac{\partial z}{\partial \mathbf{n}_{n 1}}\right]+B\left[\frac{\partial x}{\partial \mathbf{n}_{n 1}}\right]\right\}, \\
\frac{\partial H}{\partial y}\left(\boldsymbol{x}, \mathbf{n}_{n 1}, \mathbf{n}_{n 2}, \boldsymbol{x}_{0}, \omega\right)=\frac{1}{4 \pi \lambda}\left\{A\left[\frac{\partial r}{\partial x} \frac{\partial r}{\partial y} \frac{\partial x}{\partial \mathbf{n}_{n 1}}+\left(\frac{\partial r}{\partial y}\right)^{2} \frac{\partial y}{\partial \mathbf{n}_{n 1}}+\frac{\partial r}{\partial y} \frac{\partial r}{\partial z} \frac{\partial z}{\partial \mathbf{n}_{n 1}}\right]+B\left[\frac{\partial y}{\partial \mathbf{n}_{n 1}}\right]\right\}, \\
\frac{\partial H}{\partial z}\left(\boldsymbol{x}, \mathbf{n}_{n 1}, \mathbf{n}_{n 2}, \boldsymbol{x}_{0}, \omega\right)=\frac{1}{4 \pi \lambda}\left\{A\left[\frac{\partial r}{\partial x} \frac{\partial r}{\partial z} \frac{\partial x}{\partial \mathbf{n}_{n 1}}+\frac{\partial r}{\partial y} \frac{\partial r}{\partial z} \frac{\partial y}{\partial \mathbf{n}_{n 1}}+\left(\frac{\partial r}{\partial z}\right)^{2} \frac{\partial z}{\partial \mathbf{n}_{n 1}}\right]+B\left[\frac{\partial z}{\partial \mathbf{n}_{n 1}}\right]\right\}, \\
A=-\frac{k_{c}{ }^{2} \mathrm{e}^{-\mathrm{i} k_{c} r}}{r}+\frac{3 \mathrm{i} k_{c} \mathrm{e}^{-\mathrm{i} k_{c} r}}{r^{2}}+\frac{3 \mathrm{e}^{-\mathrm{i} k_{c} r}}{r^{3}} \text { and } B=-\frac{\mathrm{i} k_{c} \mathrm{e}^{-\mathrm{i} k_{c} r}}{r^{2}}-\frac{\mathrm{e}^{-\mathrm{i} k_{c} r}}{r^{3}} .
\end{gathered}
$$

In Eqn (6) the incident heat field is computed as 


$$
\bar{T}_{i n c}\left(\boldsymbol{x}, \mathbf{n}_{n 2}, \boldsymbol{x}_{s}, \omega\right)=\frac{\mathrm{e}^{-i k_{c} r_{0}}\left(-\mathrm{i} k_{c} r_{0}-1\right)}{2 \lambda r_{0}^{2}}\left(\frac{\partial r_{0}}{\partial x} \frac{\partial x}{\partial \mathbf{n}_{n 2}}+\frac{\partial r_{0}}{\partial y} \frac{\partial y}{\partial \mathbf{n}_{n 2}}+\frac{\partial r_{0}}{\partial z} \frac{\partial z}{\partial \mathbf{n}_{n 2}}\right)
$$

The global solution is found by solving Eqn (6), which requires the discretization of the interface $S$ into $N$ planar boundary elements, with one nodal point in the middle of each element.

The integrations in Eqn (6) are evaluated using a Gaussian quadrature scheme when the element to be integrated is not the loaded element. For the loaded element (the hypersingular element), however, the integrands exhibit a singularity and the integration can be carried out in closed form, as will be defined next.

Consider a singular rectangular element of width $\mathrm{W}$ (in the $x$ direction) and length $\mathrm{L}$ (in the $z$ direction) and with the axis origin at the centre of the element.

The integration of the Green's function $\int_{-L / 2}^{L / 2} \int_{-W / 2}^{W / 2} \bar{H}\left(\boldsymbol{x}, \mathbf{n}_{n 1}, \mathbf{n}_{n 2}, \boldsymbol{x}_{0}, \omega\right) d x d z$ (with $\mathbf{n}_{n 2}=\mathbf{n}_{n 1}$ ) leads to a hypersingular term. The evaluation of this integration can be performed by writing $\bar{H}\left(\boldsymbol{x}, \mathbf{n}_{n 1}, \mathbf{n}_{n 2}, \boldsymbol{x}_{0}, \omega\right)$ as the sum of two-dimensional Green's functions with varying spatial wavenumbers. This is accomplished by first applying a spatial Fourier transformation along the $z$ direction to the three dimensional Green's function $H\left(\boldsymbol{x}, \mathbf{n}_{n 1}, \boldsymbol{x}_{0}, \omega\right)$. The application of a Fouriertransformation to Eqn (5) in that direction leads to a line heat field, whose amplitude varies sinusoidally in the third dimension $(z)$,

$$
\hat{H}\left(x, y, \mathbf{n}_{n 1}, x_{0}, y_{0}, k_{z}, \omega\right)=\frac{i k}{8 \pi \lambda} H_{1}\left(k \bar{r}_{0}\right)\left(\frac{\partial \bar{r}_{0}}{\partial x} \frac{\partial x}{\partial \boldsymbol{n}_{n 1}}+\frac{\partial \bar{r}_{0}}{\partial y} \frac{\partial y}{\partial \boldsymbol{n}_{n 1}}\right),
$$

in which $H_{n}(\ldots)$ are second kind Hankel functions of the order $n$, $k=\sqrt{-\frac{\mathrm{i} \omega}{K}-k_{z}^{2}}$, with $\operatorname{Im}(k)<0, \quad \bar{r}_{0}=\sqrt{\left(x-x_{0}\right)^{2}+\left(y-y_{0}\right)^{2}}, \quad$ where $\quad k_{z} \quad$ is the wavenumber in the $z$ direction.

Assuming the presence of an infinite set of equally-spaced sources in the $z$ direction, the former Green's function can be recast as:

$$
H\left(\boldsymbol{x}, \mathbf{n}_{n 1}, \boldsymbol{x}_{0}, \omega\right)=\frac{2 \pi}{L_{v s}} \sum_{m=-\infty}^{\infty} \hat{H}\left(x, y, \mathbf{n}_{n 1}, x_{0}, y_{0}, k_{z}, \omega\right) \mathrm{e}^{-\mathrm{i} k_{z m} z},
$$

where $L_{v s}$ is the spatial source interval, and $k_{z m}=\frac{2 \pi}{L_{v s}} m$.

This equation converges and can be approximated by a finite sum of terms (M). The distance $L_{v s}$ needs to be large enough to avoid spatial contamination. The use of complex frequencies further reduces the influence of the neighbouring fictitious sources. The 3D Green's field can therefore be computed as the pressure irradiated by a sum of harmonic (steady-state) line loads, whose amplitude varies sinusoidally in the $z$ dimension.

The Green's functions $\bar{H}$ can then be defined by applying the gradient operator to $H$, which leads to 


$$
\bar{H}\left(x, y, \mathbf{n}_{n 1}, \mathbf{n}_{n 1}, x_{0}, y_{0}, k_{z}, \omega\right)=\frac{\mathrm{i} k}{8 \pi \lambda}\left[-k H_{2}\left(k \bar{r}_{0}\right)\left(\frac{\partial \bar{r}_{0}}{\partial x} \frac{\partial x}{\partial \mathbf{n}_{n l}}+\frac{\partial \bar{r}_{0}}{\partial y} \frac{\partial y}{\partial \mathbf{n}_{n l}}\right)^{2}+\frac{H_{1}\left(k \bar{r}_{0}\right)}{\bar{r}_{0}}\right],
$$

This procedure allows the integration $\int_{-L / 2}^{L / 2} \int_{-W / 2}^{W / 2} \bar{H}\left(\boldsymbol{x}, \mathbf{n}_{n 1}, \mathbf{n}_{n 2}, \boldsymbol{x}_{0}, \omega\right)$ to be obtained as

$$
\begin{gathered}
\int_{-L / 2}^{L / 2} \int_{-W / 2}^{W / 2} \bar{H}\left(\boldsymbol{x}, \mathbf{n}_{n 1}, \mathbf{n}_{n 2}, \boldsymbol{x}_{0}, \omega\right) d x d z=\frac{2 \pi}{L_{v s}} \sum_{m=-M}^{M} \int_{-L / 2}^{L / 2} I_{2}\left(k \bar{r}_{0}\right) \mathrm{e}^{-\mathrm{i} k_{z m} z} d z, \\
\text { with } \int_{-L / 2}^{L / 2} I_{2}\left(k \bar{r}_{0}\right) \mathrm{e}^{-\mathrm{i} k_{z m} z} d z=I_{2}\left(k \bar{r}_{0}\right) L \text { if } m=0 \\
\int_{-L / 2}^{L / 2} I_{2}\left(k \bar{r}_{0}\right) \mathrm{e}^{-\mathrm{i} k_{z m} z} d z=I_{2}\left(k \bar{r}_{0}\right) \frac{2 \sin \left(k_{z m} \frac{L}{2}\right)}{k_{z m}} \text { if } m \neq 0 \\
\text { where } I_{2}\left(k \bar{r}_{0}\right)=\int_{-W / 2}^{W / 2} \frac{\mathrm{i} k}{8 \pi \lambda}\left[-k H_{2}\left(k \bar{r}_{0}\right)\left(\frac{\partial \bar{r}_{0}}{\partial x} \frac{\partial x}{\partial \mathbf{n}_{n l}}+\frac{\partial \bar{r}_{0}}{\partial y} \frac{\partial y}{\partial \mathbf{n}_{n l}}\right)^{2}+\frac{H_{1}\left(k \bar{r}_{0}\right)}{\bar{r}_{0}}\right] d x
\end{gathered}
$$

The integration of $I_{2}\left(k \bar{r}_{0}\right)$ is performed indirectly by isolating a semicylinder just above the boundary element and by considering its thermal equilibrium, which leads to

$$
I_{2}\left(k \bar{r}_{0}\right)=\frac{\mathrm{i} k^{2}}{4 \pi \lambda}\left[\int_{0}^{W / 2} H_{0}\left(k \bar{r}_{0}\right) d \bar{r}_{0}-\frac{1}{k} H_{1}\left(k \frac{W}{2}\right)\right]
$$

\section{Algorithm's verification}

This section illustrates the verification of the proposed algorithm using a circular cylindrical cavity, aligned along the $z$ axis, for which analytical solutions can be derived. This cavity, with radius $a$, is excited by a point heat source placed at $\left(x_{s}, y_{s}, z_{s}\right)$. Null heat fluxes are prescribed along the boundary. To enable comparison with the 3D TBEM model, the length of the cavity is limited by enforcing null heat fluxes at sections $z=0.0 \mathrm{~m}$ and $z=L_{t}$.

The analytical solution for this problem is obtained by first applying a spatial Fourier transformation in the $z$ direction, which allows the solution to be obtained as the sum of two-dimensional solutions with a varying spatial wavenumber in that direction. The null normal heat flux at sections $z=0.0 \mathrm{~m}$ and $z=L_{t}$ are accomplished by adding the heat field generated by the real source to that produced by virtual sources (image sources), which are located in the $z$ direction in such a way that they act as mirrors of the real source and that they guarantee the required boundary conditions.

$$
T\left(\boldsymbol{x}, \boldsymbol{x}_{s}, \omega\right)=\frac{2 \pi}{L_{v s}} \sum_{m=-\infty}^{\infty} \hat{T}^{\prime}\left(x, y, x_{s}, y_{s}, k_{z m}, \omega\right),
$$


with $\hat{T}^{\prime}\left(x, y, x_{s}, y_{s}, k_{z m}, \omega\right)=\hat{T}\left(x, y, x_{s}, y_{s}, k_{z m}, \omega\right)\left[\mathrm{e}^{-\mathrm{i} k_{z m} z_{m 0}}+\sum_{m=1}^{N S_{z}} \sum_{j=1}^{4} \mathrm{e}^{-\mathrm{i} k_{z m} z_{m j}}\right]$

where $\quad z_{m 0}=z-z_{s}, \quad z_{m 1}=z+z_{0}-2 L_{t} m, \quad z_{m 2}=z+z_{0}+2 L_{t}(m-1)$, $z_{m 3}=z-z_{0}-2 L_{t} m$ and $z_{m 3}=z-z_{0}+2 L_{t} m$.

The number of virtual sources $N S_{z}$ to be used in the calculations is defined so that the signal responses can be correctly computed in the time frame, which is determined by the frequency increment $1 / \Delta f$. This procedure does not introduce any type of error into the computed time impulse response within the time window defined. Notice that $L_{v s}$ should be at least twice the distance from the real source to the more distant virtual source. Each two-dimensional problem is solved using the separation of variables procedure with the Helmholtz equation and enforcing the boundary conditions throughout the boundary surface, using the Bessel series form. The following equations can be derived if the origin of the coordinate system coincides with the centre of the circle, cross section of the cylinder, and the source lies on the $x$ axis.

$$
\hat{T}\left(x, y, x_{s}, y_{s}, k_{z}, \omega\right)=-\frac{\mathrm{i}}{4 \lambda} H_{0}\left(k \overline{r_{0}}\right)+\sum_{n=0}^{\infty} A_{n} H_{n}(k \bar{r}) \cos (n \theta),
$$

with $A_{n}=\frac{\frac{\mathrm{i}}{4 \lambda}(-1)^{n} \varepsilon_{n} H_{n}\left(k \bar{r}_{00}\right)\left[\frac{n}{a} J_{n}(k a)-k J_{n+1}(k a)\right]}{\left[\frac{n}{a} H_{n}(k a)-k H_{n+1}(k a)\right]}, \bar{r}=\sqrt{x^{2}+y^{2}}, J_{n}(\ldots)$ are

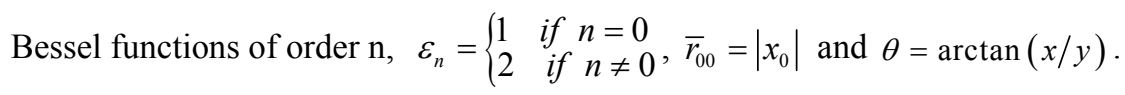

The temperature responses are computed at $(-0.8 \mathrm{~m}, 0.0 \mathrm{~m}, 1.0 \mathrm{~m})$, in the vicinity of a cylinder inclusion with a radius of $a=0.5 \mathrm{~m}$ and $L_{t}=2.0 \mathrm{~m}$. The $3 \mathrm{D}$ heat source is placed at $(-1.5 \mathrm{~m}, 0.0 \mathrm{~m}, 1.0 \mathrm{~m})$. Computations are performed with a frequency increment of $0.5 \times 10^{-7} \mathrm{~Hz}$ in the frequency range $\left[0.0,1 \times 10^{-5}\right] \mathrm{Hz}$. The thermal properties assigned to the medium are: $c=780 \mathrm{~J} \cdot \mathrm{kg}^{-1} \cdot{ }^{\circ} \mathrm{C}^{-1}, \rho=1860 \mathrm{~kg} \cdot \mathrm{m}^{-3}$ and $\lambda=0.72 \mathrm{~W} \cdot \mathrm{m}^{-1} \cdot{ }^{\circ} \mathrm{C}^{-1} \cdot L_{v s}$ is assumed to be $60.0 \mathrm{~m}$. The responses were computed for complex frequencies $\omega_{c}=\omega-\mathrm{i} \eta$ (with $\eta=0.7 \Delta \omega, \Delta \omega=2 \times \pi \times \Delta f$ and $\Delta f=0.5 \times 10^{-7} \mathrm{~Hz}$ ).

The responses were calculated both analytically and by using the TBEM. To understand the behaviour of the various solutions two different numbers of boundary elements were used to discretise the inclusion: $30 \times 20(20$ in the $z$ direction) and $50 \times 32$ (32 in the $z$ direction).

Figure 2 shows the analytical response (real and imaginary parts) at the receiver and illustrates the value of the error when the problem is solved using the proposed TBEM algorithm. It can be observed that the solution improves as the number of boundary elements increases. 

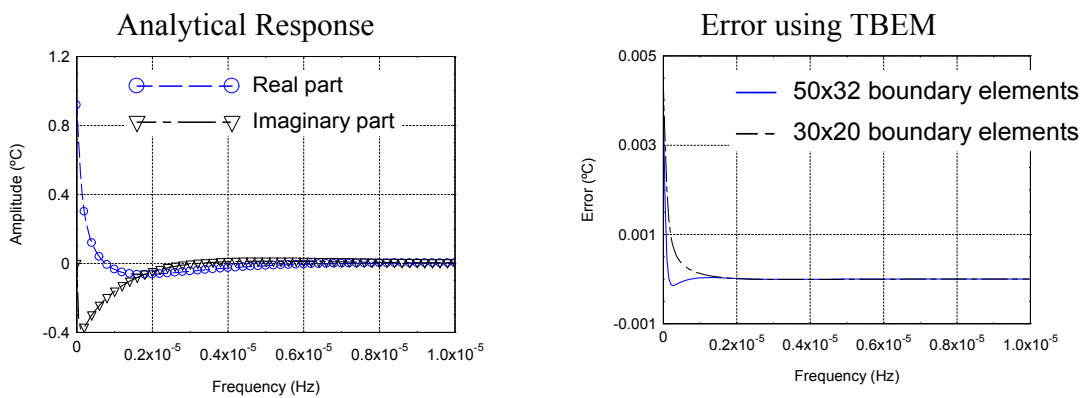

Figure 2: Comparative analysis of analytical and TBEM responses, considering different numbers of boundary elements.

\section{Temperature in time}

A numerical inverse fast Fourier transform is applied in the frequency domain to find the heat field in the time domain. Aliasing phenomena are dealt by introducing complex frequencies with a small imaginary part, taking the form $\omega_{c}=\omega-\mathrm{i} \eta$ (where $\eta=0.7 \Delta \omega$, and $\Delta \omega$ is the frequency step). This shift is subsequently taken into account in the time domain by means of an exponential window, $\mathrm{e}^{\eta t}$, applied to the response.

The source can have any time variation. We can determine the frequency domain solution by applying a time Fourier transformation, and it can range from $0.0 \mathrm{~Hz}$ to quite high frequencies. Since the heat response falls rapidly with increasing frequency, we do not need to compute the highest frequencies in the range.

\section{Simulation results}

The applicability and usefulness of the proposed approach are illustrated by simulating the heat propagation around a 3D thin crack hosted in an unbounded solid medium. The cracks, modelled next, are assumed to be plan, rectangular and placed parallel to the $z$ axis. Its size, orientation and position vary (see Figure 3 ). The orientation of the crack is changed by tilting the crack. Two crack sizes are simulated: $(a=0.1 \mathrm{~m}, b=0.2 \mathrm{~m})$ and $(a=0.05 \mathrm{~m}, b=0.1 \mathrm{~m})$.

The thermal properties of the host medium are those of the concrete, mentioned above. All the calculations are performed in the frequency range $\left[0.0,1.42 \times 10^{-2}\right] \mathrm{Hz}$ with a frequency increment of $\Delta \omega=2.778 \times 10^{-5} \mathrm{~Hz}$ and the imaginary part of the frequency is given by $\eta=0.7 \Delta \omega$.

The computations are performed for the following cases: Case I) grid of receivers are placed at $x=0.08 \mathrm{~m}, x=0.22 \mathrm{~m}$ and $z=0.0 \mathrm{~m}, 3 \mathrm{D}$ source point is placed at $(0.0 \mathrm{~m}, 0.0 \mathrm{~m}, 0.0 \mathrm{~m})$ and vertical crack is $0.1 \times 0.2 \mathrm{~m}^{2}$; Case II) 


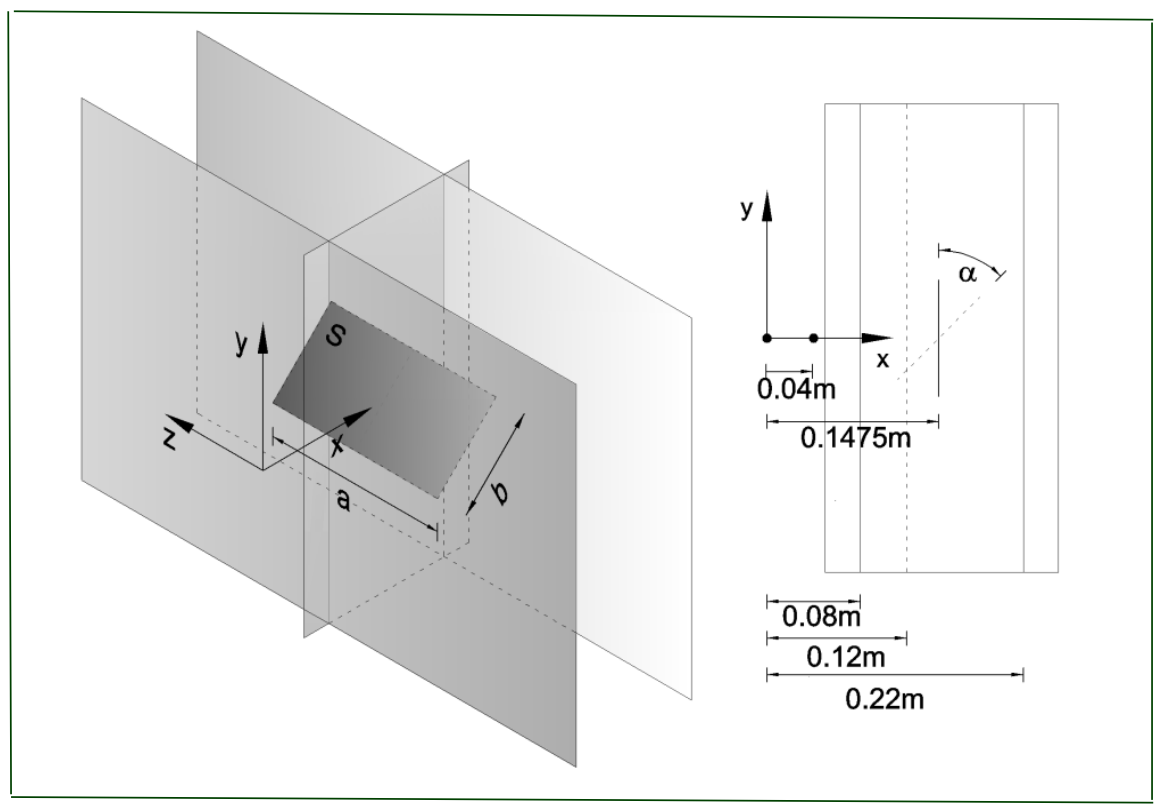

Figure 3: Geometry of the system used to simulate infrared thermography.

same as before but crack is tilted at $45^{\circ}$; Case III) receivers are placed at $x=0.12 \mathrm{~m}, \quad x=0.22 \mathrm{~m}$ and $z=0.0 \mathrm{~m}$, source point is at $(0.04 \mathrm{~m}, 0.0 \mathrm{~m}, 0.0 \mathrm{~m})$ and $0.1 \times 0.2 \mathrm{~m}^{2}$ vertical crack; Case IV) same as Case III but crack is reduced to $0.05 \times 0.1 \mathrm{~m}^{2}$. On each grid, receivers were spaced at equal intervals of $0.005 \mathrm{~m}, 0.00625 \mathrm{~m}$ and $0.006 \mathrm{~m}$ in the $x, y$, and $z$ axis direction, respectively.

The host medium is assumed to be at $20.0^{\circ} \mathrm{C}$ when the source starts emitting energy. The source time dependence is assumed to be rectangular with a power of 12 Watts. It starts emitting energy at instant $t=0.5 \mathrm{~h}$ and continues for $1.5 \mathrm{~h}$. The grid of receivers placed at $x=0.08 \mathrm{~m}$ or $x=0.12 \mathrm{~m}$ simulates an object's surface on which thermo graphic data given by IRT is recorded.

By comparing Cases I and II the influence of the crack's inclination on the temperatures results recorded on the grid of receivers is studied. Case III evaluates the alterations produced when the crack is moved closer to the object's surface and finally Case IV studies the effect of reducing the size of the crack.

In order to study the effect of the crack on the temperature pattern, computations were made for when the crack is embedded in the medium and for when the medium is empty of cracks. The difference between both results translates the differences in temperatures that may be used by IRT to assess the presence of cracks. This procedure is exemplified in Figure 4, which illustrates 

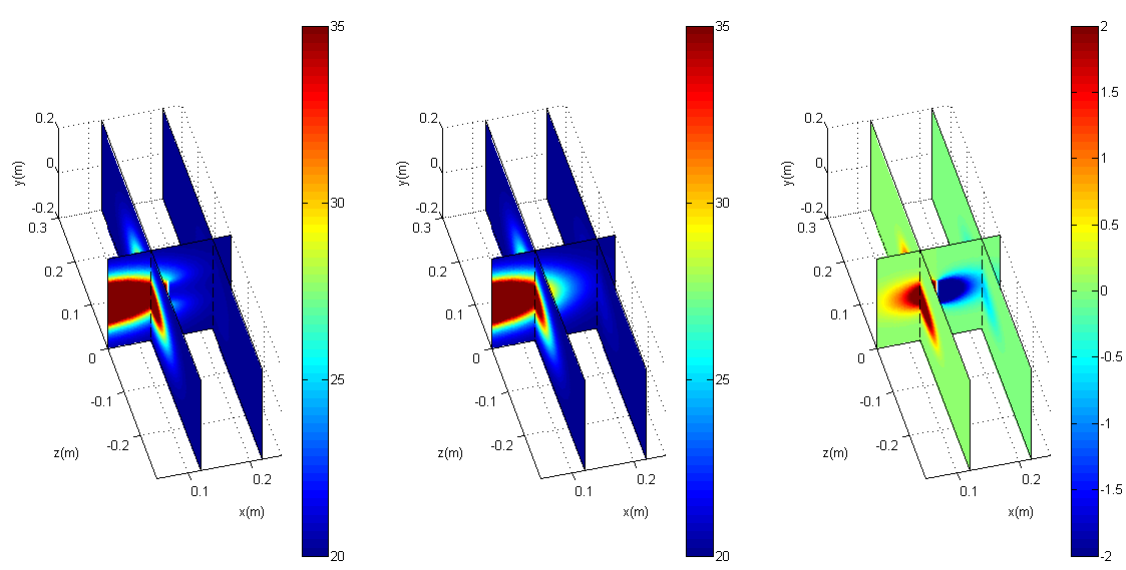

Figure 4: $\quad$ Snapshots of temperature distribution $\left(\right.$ in ${ }^{\circ} \mathrm{C}$ ) for Case III.

the time domain snapshots of the temperature distribution at $t=2.15 \mathrm{~h}$ for Case III. On the left, the results display the case for when the crack is within the medium, while the central plot shows the results obtained when the medium is empty of cracks. The difference between both results is given in the plot on the right.

Figure 5 contains snapshots of the temperature differences at different time instants $(t=1.5 \mathrm{~h}, t=2.15 \mathrm{~h}$ and $t=4.5 \mathrm{~h})$ for each of the cases described previously. The scale of colours shown in the plot on the right in Figure 4 also applies to Figure 5. As shown in Figure 5, given the distance from the crack to the grid of receivers on $x=0.08 \mathrm{~m}$, the temperature field is very mild for both cases (I and II). This field is progressively weaker as the crack is tilted. Ultimately, when the crack is tilted horizontally the temperature difference is null, due to fact that the crack is aligned with the point heat source $(z=0)$ In this particular case, the heat front is perpendicular to the crack, so null fluxes exist for any azimuthal directions. Thus, the crack does not introduce any disturbance on the heat diffusion phenomenon. Additionally, the results show that at $t=1.5 \mathrm{~h}$ the temperature is still increasing, while at $t=4.5 \mathrm{~h}$ the source has already been turned off and the temperature is decreasing. The temperature field recorded on the perpendicular grid $(z=0.0 \mathrm{~m})$ witnesses this behaviour.

Results for case III show that by bringing the crack closer to the surface, the temperature field becomes more significant, when compared to cases I and II. The results obtained by reducing the size of the crack illustrates that the effect that the inclusion has on the surface temperature differences diminishes. In fact, temperature differences of this scale are not picked up by most IRT systems. This indicates that further studies need to be performed by using a more powerful heat source or by changing the excitation frequency and performing more than one heating cycle. 

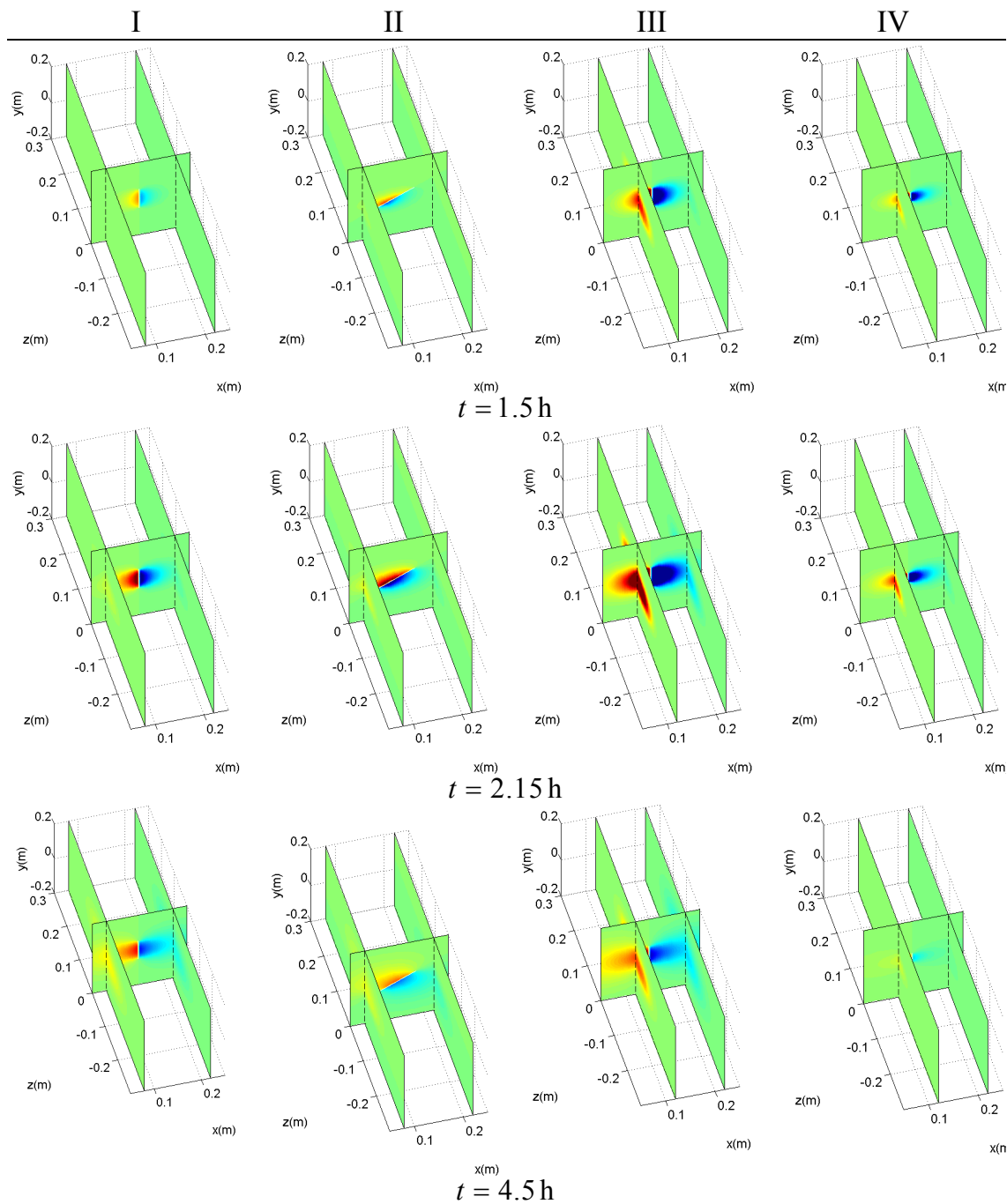

Figure 5: $\quad$ Snapshots of temperature differences.

\section{Conclusions}

In this paper the authors proposed a three-dimensional normal-derivative integral equation (TBEM) formulation to allow modelling heat conduction in the vicinity of cracks embedded in an unbounded medium. The integration of hypersingular integrals that appear when the element to be integrated is the loaded one (singular element) are integrated analytically. 
The proposed algorithm was first verified against a reference solution defined for a circular cylindrical cavity and very good accuracy was demonstrated. Finally, the proposed coupling formulations were applied to a set of numerical examples where a crack is placed in an unbounded space. The size of the crack, its orientation and the position of the source were some of the variables that are analyzed to define features that may be used in IRT.

\section{Acknowledgements}

The research work presented herein was supported by FEDER funds through the Operational Programme for Competitiveness Factors - COMPETE and by national funds through the FCT - Portuguese Foundation for Science and Technology), under research project PTDC/ECM/114189/2009. The presented work is also framed under the Energy for Sustainability Initiative of the University of Coimbra, through the MIT PORTUGAL, supported by the FCT.

\section{References}

[1] Grinzato, E, V Vavilov, and T Kauppinen. Quantitative infrared thermography in buildings. Image Processing 29. 1998.

[2] Balaras, C A, and A A Argiriou. Infrared thermography for building diagnostics. Energy and Buildings 2002. 34: 171-183.

[3] Sham FC, Chen N, Long L. Surface crack detection by flash thermography on concrete surface. Insight 2008; 50(5):240-243.

[4] Maierhofer Ch, Arndt R, Rollig M. Influence of concrete properties on the detection of voids with impulse-thermography. Infrared Physics and Technology 2007; 49:213-217.

[5] Maierhofer, Ch., H. Wiggenhauser, a. Brink, and M. Röllig. Quantitative numerical analysis of transient IR-experiments on buildings. Infrared Physics \& Technology 2004; 46 (1-2): 173-180.

[6] Brebbia CA, Telles JC, Wrobel LC. Boundary Elements Techniques: Theory and Applications in Engineering. Springer-Verlag, Berlin-New York, 1984.

[7] Rudolphi TJ. The use of simple solutions in the regularisation of hypersingular boundary integral equations. Mathematical and Computer Modelling 1991; 15: 269-278.

[8] Cruse TA. Boundary Element Analysis in Computational Fracture Mechanics. Kluwer Academic Publishers, 1987.

[9] Sládek V, Sládek J. Transient elastodynamics three-dimensional problems in cracked bodies. Applied Mathematical Modelling 1984; 8: 2-10.

[10] Amado Mendes P, Tadeu A. Wave propagation in the presence of empty cracks in an elastic medium. Computational Mechanics 2006; 38(3): 183199.

[11] Srivastava DN. Contractor, Efficient evaluation of integrals in 3-D Boundary Element Method using linear shape functions over plane triangular elements. Applied Mathematical Modelling 1992; 16(6): 282-290. 